\title{
Oscillometric blood pressure by age and height for non overweight children and adolescents in Lubumbashi, Democratic Republic of Congo
}

Emmanuel Kiyana Muyumba', Dophra Ngoy Nkulu', Clarence Kaut Mukeng², Jacques Mbaz Musung ${ }^{3}$, Placide Kambola Kakoma', Christian Ngama Kakisingi ${ }^{1}$, Oscar Numbi Luboya ${ }^{4,5,6}$, Françoise Kaj Malonga ${ }^{5}$, Justin Kalungwe Kizonde ${ }^{7}$, Olivier Mukuku, ${ }^{4,6^{*}}$ and Weili Yan ${ }^{8}$

\begin{abstract}
Background: The diagnosis of hypertension in children is complex because based on normative values by sex, age and height, and these values vary depending on the environment. Available BP references used, because of the absence of local data, do not correspond to our pediatric population. Accordingly, our study aimed to provide the BP threshold for children and adolescents in Lubumbashi (DRC) and to compare them with German (KIGGS study), Polish (OLAF study) and Chinese (CHNS study) references.

Methods: We conducted a cross-sectional study among 7523 school-children aged 3 to 17 years. The standardized BP measurements were obtained using a validated oscillometric device (Datascope Accutor Plus). After excluding overweight and obese subjects according to the IOTF definition $(n=640)$, gender-specific SBP and DBP percentiles, which simultaneously accounted for age and height by using an extension of the LMS method, namely GAMLSS, were tabulated.

Results: The 50th, 90th and 95th percentiles of SBP and DBP for 3373 boys and 3510 girls were tabulated simultaneously by age and height (5th, 25th, 50th, 75th and 95th height percentile).

Before 13 years the 50th and 90th percentiles of SBP for boys were higher compared with those of KIGGS and OLAF, and after they became lower: the difference for adolescents aged 17 years was respectively $8 \mathrm{mmHg}$ (KIGGS) and $4 \mathrm{mmHg}$ (OLAF). Concerning girls, the SBP 50th percentile was close to that of OLAF and KIGGS studies with differences that did not exceed $3 \mathrm{mmHg}$; whereas the 90th percentile of girls at different ages was high. Our oscillometric 50th and 90th percentiles of SBP and DBP were very high compared to referential ausculatory percentiles of the CHNS study respectively for boys from 8 to $14 \mathrm{mmHg}$ and 7 to $13 \mathrm{mmHg}$; and for girls from 10 to $16 \mathrm{mmHg}$ and 11 to $16 \mathrm{mmHg}$.
\end{abstract}

Conclusions: The proposed BP thresholds percentiles enable early detection and treatment of children and adolescents with high BP and develop a local program of health promotion in schools and family.

Keywords: Blood pressure, Children, Adolescents, Lubumbashi, Percentile tables, GMLSS, LMS

\footnotetext{
* Correspondence: oliviermukuku@yahoo.fr

${ }^{4}$ Department of Pediatrics, University Clinic, University of Lubumbashi,

Lubumbashi, Democratic Republic of Congo

${ }^{6}$ Department of Research, Higher Institute of Medical Techniques,

Lubumbashi, Democratic Republic of Congo

Full list of author information is available at the end of the article
} 


\section{Background}

Several publications have shown the importance of measuring the blood pressure (BP) and hypertension in childhood and adolescence [1-4]. In children and adolescents, high $\mathrm{BP}$ values are associated with left ventricular hypertrophy [5], increase in thickness intima-media of arteries [6] and they are predictive of hypertension in adulthood [7].

Pediatricians have at their disposal BP references tables, which determine whether $\mathrm{BP}$ is normal or if it is a threshold that requires the application of the assessment, prevention or treatment. BP references are based on sex, age and height, but also on study populations characteristics such as ethnicity or nationality, including the type of device used to measure BP $[1,2,8,9]$.

Obesity has become epidemic in the world in adults as well as in children and adolescents $[10,11]$. Adiposity in childhood, as measured by body mass index (BMI) [12] is an important predictor of elevated BP. In a study among 197,191 children aged 7-17 years obtained from a Chinese national survey in 2010, Dong et al. [13] noted that overweight and obese children have a significantly higher risk of high BP than non-overweight children.

$\mathrm{BP}$ references or standards are not available for Congolese children in the Democratic Republic of Congo (DRC) but there are many others $[1,2,14-16]$ that do not correspond to our pediatric population.

The objectives of our study were to establish the BP threshold percentiles of non-overweight children and adolescents in Lubumbashi (DRC) and compare them with German, Polish and Chinese BP references.

\section{Methods}

\section{Design, participants and setting of the study}

The study took place in Lubumbashi (Province of HautKatanga) the second city of the DRC by its economic, political, social and cultural importance. The studied population was composed of school children aged 3 to 17 years, enrolled in pre-primary, primary and secondary schools during the school-years 2013-2014, 2014-2015 and 2015-2016.

A cross-sectional study was conducted on a representative sample of these pupils, who were recruited in randomly selected clusters using two-stage sampling. The first cluster was comprised of selected schools from all private and public schools of Lubumbashi. The second cluster was comprised of randomly selected classes within each of the selected schools. All pupils in the selected classes were invited to join the study. Sampling was stratified by township.

For a strate (township), the equation allowing the calculation of the sample was the following:

$n=z^{2} \frac{p(1-p)}{e^{2}}$

In which: $-\mathrm{z}$ is the level of confidence $(=95 \%)$. $-p$ is the initial level of the school attendance rate $(=50 \%)$.

- $e$ is the error margin $(=0.05 \%)$.

The size calculated in this way had been adjusted according to the effect of the sampling plan $(=1.5 \%)$, the number of estimations by age group and by sex (= 3\%) and this was reported to the expected number of nonanswers $(=20 \%)$. The number thus obtained was multiplied by the number of the strate (township) of the city of Lubumbashi (for details, please consult website www.unilu.ac.cd/wp-content/uploads/2016/07/

Seuils1.pdf).

Informed written consent was obtained from parents or guardians. The approval to conduct the study and authorizations were obtained from the Medical Ethic Committee of the University of Lubumbashi (UNILU/ CEM/027/2013-27 september 2013), the Provincial Ministry of Education, Scientific Research, Transport and Energy of Katanga ( $\mathrm{N}^{\circ} 10.5 / 0209 / \mathrm{CAB} / \mathrm{MIN} . \mathrm{PROV} /$ ED.R.TE/KAT/2014-11 march 2014) and the authorities of the selected schools.

Data were collected by trained medical staff. The study team received refresher training at the beginning of each data collection phase.

\section{BP measurement}

BP was measured by using a Datascope Accutorr Plus (Datascope Corporation, USA). The appropriate cuff size (bladder width at least $40 \%$ of arm circumference and length to cover $80-100 \%$ of arm circumference) was determined by measuring the mid-upper arm circumference.

$\mathrm{BP}$ measurements were performed at least $30 \mathrm{~min}$ after exercise or the last meal, in a subject at rest 5 min before setting, in a seated position with arm and back supported, feet resting on the floor and legs uncrossed. The cuff was applied to the right arm, at the heart level, then wrapped in a sealing which did not allow two fingers to be inserted under it. The lower edge of the cuff was placed $2 \mathrm{~cm}$ from the cubital fossa. Three readings were obtained at a $1 \mathrm{~min}$ intervals on the same day and the mean of the second and third readings was used for analysis.

\section{Anthropometric measurements}

Body weight was measured in duplicate with the participant wearing light underwear, barefoot, standing on a digital scale. Body weight was recorded nearest $0.1 \mathrm{~kg}$. Body height recorded nearest $0.1 \mathrm{~cm}$ was measured in duplicate. Each participant was in standing upright position, barefoot with shoulders and hips perpendicular at the central axis, heels against the step, knees together, arms relaxed along the body and head straight. Special attention was given to children (under 6 years) using a second investigator to block the movement of the knees. The mean of the two measurements of weight and height and were selected for statistical analysis. 
BMI was calculated as weight (in kilograms) divided by the square of height (in meters). The terms overweight and obese were defined according to the International Obesity Task Force (IOTF) definition [17].

\section{Inclusion and exclusion criteria for the sample on which the percentiles are based}

Of 8371 participants consented, we excluded 1488: 795 children were younger than 3 years $(n=55)$ or over 17 years $(n=740), 53$ had outlying or missing data (date of birth, BP, weight, height) and 640 were recorded overweight $(n=548)$ or obese $(n=92)$. No child had been reported to have a chronic disease likely to influence weight or blood pressure and also no child was taking a medicine having an influence on the blood pressure (Additional file 1).

\section{Statistical analysis}

All statistical analyses were performed using SPSS version 22.0 (SPSS Inc., Chicago, IL, USA) and the free statistical software R 3.1.2 (2014-10-31) (http://www.cran.rproject.org).

Thresholds of BP by gender were constructed by age and height simultaneously using an extension of the LMS method [18], namely generalized additive models for location scale and shape (GAMLSS) with the Box-Cox power exponential (BCPE) and Box-Cox-Cole-Green (BCCG) distributions families, fitted with GAMLSS 4.3-1 in the free statistical software R 3.1.2 (2014-10-31). In GAMLSS [18, 19], four parameters $(\mu, \sigma, v, \tau)$ were used to define the location, scale and shape of the BP distribution with age and height. To obtain the optimal models by minimizing the Schwarz Bayesian Criterion [20], linear and additive effect of age and height on systolic blood pressure (SBP) and diastolic blood pressure (DBP) were modeled simultaneously. The threshold values of the 50th, 90th and 95th percentiles of SBP and DBP were calculated by age and height (exact heights according to the 5th, 25th, 50th, 75th and 95th percentiles) for boys and girls separately.

Different percentiles of SBP and DBP (50th and 90th percentiles) for boys and girls, were compared with references percentiles of the German Health Interview and Examination Survey for Children and Adolescents (KIGGS) [14], the Elaboration of the Ranks of Reference Arterial Blood Pressure for the Population of Children and Adolescents in Poland (OLAF) [15] and the Chinese reference that used data from the China Health and Nutrition Survey (CHNS) [16]. This comparison was made for the different ages in relation to the target population of these studies (3-17 years for KIGGS and 7-17 years for OLAF and CHNS).

\section{Results}

Of the 11,283 pupils selected and invited to take part in the study, 8371 children and adolescents consented and were enrolled for a global participation rate of $74.2 \%$. Data were collected three times (according to the school calendar in the DRC) from March 2014 to December 2015; in 66 schools. The reference population of nonoverweight children and adolescents aged 3 to 17 years consisted of 3373 boys and 3510 girls.

Table 1 summarized the baseline characteristics of 6883 non-overweight children and adolescents. The mean of SBP and DBP increased with age in both sexes. The mean of the first BP (both systolic and diastolic) was the highest and the mean of the third BP was the lowest in the series of three measures in both sexes and in all age groups. The mean of the first and second BP was higher: 0.8 to $1.4 \mathrm{mmHg}$ SBP and 0.9 to $2.0 \mathrm{mmHg}$ DBP, in comparison with the average of the second and third BP (Table 1).

Among the best fitted models for the 4 parameters of distribution of SBP and DBP, the Box-Cox and Cole Green (BCCG) was shown as the best fit model of SBP for both genders and DBP for girls; while the Box-Cox Power Exponential (BCPE) was for the boys' DBP.

The thresholds 50th, 90th and 95th percentiles of SBP and DBP were tabulated simultaneously by age and exact height (5th, 25th, 50th, 75th and 95th height percentile) respectively for boys and girls and are shown in Tables 2 (for boys) and 3 (for girls). The BP increased both in relation to age and height in both sexes. The median SBP and DBP were higher (about $2 \mathrm{mmHg}$ ) in girls up to the age 14 years, after this age they were almost similar in both sexes. As an illustration, in adolescents aged 17 years with a median height (167 cm for boys and $162 \mathrm{~cm}$ for girls), the median percentiles of SBP and DBP were similar, respectively $115 \mathrm{mmHg}$ and $68 \mathrm{mmHg}$. The 95 percentile used to define hypertension in children and adolescents had varied between the 5th and 95th percentile height (SBP in boys: $4-7 \mathrm{mmHg}$ and in girls: $5-8 \mathrm{mmHg}$; DBP in boys: $1-3 \mathrm{mmHg}$ and in girls: $1-2 \mathrm{mmHg}$ ).

For adolescents boys aged 11-17 years and girls aged 10-17 years, the 90th SBP percentile for the median height was respectively $120-132 \mathrm{mmHg}$ and 120-131 mmHg.

In the case of boys aged 5-12 years, the 50th SBP percentile of our oscillometric threshold was similar to the corresponding percentile of German oscillometric reference (KIGGS study), whereas compared with the Polish oscillometric reference (OLAF study) the difference were less than $2 \mathrm{mmHg}$ in age 7, 8 and 13, 14 years (Fig. 1). The 50th percentile of SBP of the KIGGS study was higher for boys $>14$ years $(8 \mathrm{mmHg}$ for adolescents aged 17 years). With regard to girls, the 50th SBP percentile of our study was close to the corresponding percentile of the KIGGS and OLAF studies: the differences did not exceed $2 \mathrm{mmHg}$, except among girls aged 9 and 10 years (for OLAF study) in which the difference was greater than $3 \mathrm{mmHg}$ (Fig. 2). 
Table 1 Baseline characteristics of children and adolescents of normal weight aged 3 to 17 years in Lubumbashi, DRC

\begin{tabular}{|c|c|c|c|c|}
\hline & \multicolumn{4}{|l|}{ Age, year } \\
\hline & $3-6$ & $7-10$ & $11-13$ & $14-17$ \\
\hline \multicolumn{5}{|c|}{ n, included (\% 3373 boys, 3510 girls) } \\
\hline Boys & $480(14.2)$ & $1161(34.4)$ & $995(29.5)$ & $737(21.9)$ \\
\hline Girls & $393(11.2)$ & $1133(32.3)$ & $1014(28.9)$ & $970(27.6)$ \\
\hline \multicolumn{5}{|c|}{ Weight, mean (SD), kg } \\
\hline Boys & $18.7(3.1)$ & $29.7(4.8)$ & $35.9(6.5)$ & $50.9(8.8)$ \\
\hline Girls & $18.6(3.5)$ & $27.1(5.5)$ & $39.0(7.5)$ & $50.4(6.5)$ \\
\hline \multicolumn{5}{|c|}{ Height, median (SD), cm } \\
\hline Boys & $111.1(8.8)$ & $130.5(9.0)$ & $142.3(9.4)$ & $162.3(10.2)$ \\
\hline Girls & $111.3(10.2)$ & $131.2(9.9)$ & $148.0(9.6)$ & $159.0(7.4)$ \\
\hline \multicolumn{5}{|c|}{$I M C$, mean $(S D) \mathrm{kg} / \mathrm{m}^{2}$} \\
\hline Boys & $15.1(1.2)$ & $15.6(1.4)$ & $16.9(1.7)$ & $19.2(2.1)$ \\
\hline Girls & $14.9(1.1)$ & $15.6(1.5)$ & $17.7(2.1)$ & $19.9(2.1)$ \\
\hline \multicolumn{5}{|c|}{ First SBP, mean (SD), mmHg } \\
\hline Boys & $101.7(11.0)$ & $104.6(10.6)$ & $109.2(10.3)$ & $118.0(12.4)$ \\
\hline Girls & $102.0(11.1)$ & $106.7(10.7)$ & $112.6(11.9)$ & $116.9(11.3)$ \\
\hline \multicolumn{5}{|c|}{ Second SBP, mean (SD), mmHg } \\
\hline Boys & $100.7(11.0)$ & $103.4(10.7)$ & $107.7(10.5)$ & $116.7(12.3)$ \\
\hline Girls & $100.3(10.6)$ & $105.5(10.7)$ & $111.2(11.1)$ & $115.5(10.8)$ \\
\hline \multicolumn{5}{|c|}{ Third SBP, mean (SD), mmHg } \\
\hline Boys & $99.7(10.9)$ & $102.4(10.0)$ & $106.8(9.9)$ & $115.3(11.9)$ \\
\hline Girls & $99.4(10.3)$ & $104.1(10.2)$ & $110.1(10.8)$ & $114.1(10.6)$ \\
\hline \multicolumn{5}{|c|}{ Mean of first and second SBP, mean (SD), mmHg } \\
\hline Boys & $101.2(10.4)$ & $104.0(9.9)$ & $108.5(9.7)$ & $117.4(11.7)$ \\
\hline Girls & $101.2(10.2)$ & $106.1(10.1)$ & $111.9(10.8)$ & $116.2(10.5)$ \\
\hline \multicolumn{5}{|c|}{ Mean of second and third SBP, mean (SD), $\mathrm{mmHg}$} \\
\hline Boys & $100.2(10.4)$ & $102.9(9.8)$ & $107.2(9.7)$ & $116.0(11.6)$ \\
\hline Girls & $99.8(9.8)$ & $104.8(10.0)$ & $110.6(10.4)$ & $114.8(10.2)$ \\
\hline \multicolumn{5}{|c|}{ First DBP, mean (SD), mmHg } \\
\hline Boys & $62.8(9.6)$ & $65.1(9.1)$ & $67.1(7.9)$ & $69.4(9.0)$ \\
\hline Girls & $63.5(9.5)$ & $66.2(9.2)$ & $68.2(8.8)$ & $70.5(8.5)$ \\
\hline \multicolumn{5}{|c|}{ Second SBP, mean (SD), mmHg } \\
\hline Boys & $61.8(8.5)$ & $63.4(9.0)$ & $65.4(8.4)$ & $67.5(8.8)$ \\
\hline Girls & $61.9(9.6)$ & $65.0(8.7)$ & $66.4(8.7)$ & $68.1(8.5)$ \\
\hline \multicolumn{5}{|c|}{ Third DBP, mean (SD), mmHg } \\
\hline Boys & $61.0(8.9)$ & $62.2(8.9)$ & $64.2(8.1)$ & $67.0(9.2)$ \\
\hline Girls & $60.9(8.8)$ & $63.7(8.9)$ & $65.2(9.0)$ & $67.0(8.5)$ \\
\hline \multicolumn{5}{|c|}{ Mean of first and second DBP, mean (SD), mmHg } \\
\hline Boys & $62.3(8.0)$ & $64.3(8.0)$ & $66.3(7.2)$ & $68.4(7.9)$ \\
\hline Girls & $62.7(8.5)$ & $65.8(7.8)$ & $67.3(7.7)$ & $69.3(7.6)$ \\
\hline \multicolumn{5}{|c|}{ Mean of second and third DBP, mean (SD), $\mathrm{mmHg}$} \\
\hline Boys & $61.4(7.7)$ & $62.8(7.9)$ & $64.8(7.4)$ & $67.3(8.1)$ \\
\hline Girls & $61.4(8.1)$ & $64.3(7.8)$ & 65.8 (7.9) & $67.6(7.6)$ \\
\hline
\end{tabular}

$S D$ standard deviation, $B M I$ body mass index, $S B P$ systolic blood pressure, $D B P$ diastolic blood pressure
For boys $<13$ years the 90th SBP percentile was higher from 1 to $4 \mathrm{mmHg}$ in comparison to KIGGS and OLAF percentiles. After, it became progressively lower: for adolescents aged 17 the difference was $8 \mathrm{mmHg}$ with the KIGGS study and $4 \mathrm{mmHg}$ with the OLAF study (Fig. 1). In the case of girls, our 90th SBP percentile was consistently higher compared to KIGGS and OLAF percentiles (Fig. 2).

The 50th and 90th DBP percentiles of the KIGGS boys (Fig. 3) were higher at all ages (among adolescents aged 17 years the difference was $4 \mathrm{mmHg}$ ). In case of the girls age range 7-17 years, the 50th and 90th DBP percentiles of OLAF study were higher when compared with our corresponding percentile.

In comparing the 50th and 90th SBP percentiles for median height for our oscillometric values to the Chinese ausculatory referential (CHNS) values, the 50th and 90th percentiles were consistently very higher among the children and adolescents of the same age: the difference was 8 to $14 \mathrm{mmHg}$ and 7 to $13 \mathrm{mmHg}$ for boys (Fig. 1); and 10 to $16 \mathrm{mmHg}$ and $11-16 \mathrm{mmHg}$ for girls (Fig. 2). The 50th and 90th DBP percentiles for boys (Fig. 3) and girls (Fig. 4) of our study were higher before age 11 years in comparison to the CHNS study; and in the age range 11-17 years, the difference were relatively minor (not exceeding $2 \mathrm{mmHg}$ ).

\section{Discussion}

This study presents the first BP threshold percentiles for children and adolescents of normal weight aged 3-17 years in Lubumbashi (DRC), computed by age and height simultaneously, by using an improved statistical method named GAMLSS provided in the package of $R$ software $[14,16,19]$. The BP percentiles were established on the basis of oscillometric measurements of the BP with a device clinically validated in children: the Datascope Accutorr Plus [21]. This device has also been used in several large studies related to $\mathrm{BP}$ in children and adolescents worldwide [14, 15, 22]. In addition, the oscillometric BP measurement is increasingly used in pediatric clinical practice.

We have not included overweight or obese subjectsin our study. Using a sample of normal weight to develop percentile allows our proposed BP thresholds to be more sensitive to the identification of children and adolescents with high BP because we avoided certain risk factors associated with the BP as being overweight or obese $[10,13]$. Sorof et al. [23] found a strong correlation between the BP and overweight and obesity.

Our mean SBP (for girls) and DBP (for boys and girls) were higher compared to those observed by Kulaga et al. [15]. Compared to the mean of the first and second BP (both systolic and diastolic) values were higher than those of children and adolescents in the KIGGS study [14]. 
Table 2 Age-height-specific thresholds: 50th, 90th and 95th percentiles of SBP and DBP values for boys aged 3-17 years

\begin{tabular}{|c|c|c|c|c|c|c|c|c|c|c|}
\hline \multirow{2}{*}{$\begin{array}{l}\text { Age, } \\
\text { year }\end{array}$} & \multirow{2}{*}{$\begin{array}{l}\text { Height, } \\
\mathrm{cm}\end{array}$} & \multirow{2}{*}{$\begin{array}{l}\text { Height } \\
\text { percentile }\end{array}$} & \multicolumn{4}{|c|}{$\mathrm{SBP}, \mathrm{mmHg}$} & \multicolumn{4}{|c|}{$\mathrm{DBP}, \mathrm{mmHg}$} \\
\hline & & & $\mathrm{S}^{*}$ & $\begin{array}{l}\text { 50th } \\
\text { percentile } \\
\text { (median) }\end{array}$ & $\begin{array}{l}\text { 90th } \\
\text { percentile }\end{array}$ & $\begin{array}{l}\text { 95th } \\
\text { percentile }\end{array}$ & $\mathrm{S}^{*}$ & $\begin{array}{l}\text { 50th } \\
\text { percentile } \\
\text { (median) }\end{array}$ & $\begin{array}{l}\text { 90th } \\
\text { percentile }\end{array}$ & $\begin{array}{l}\text { 95th } \\
\text { percentile }\end{array}$ \\
\hline \multirow[t]{5}{*}{3} & 87 & 5 th & 0.0983 & 91 & 103 & 107 & 0.0712 & 59 & 68 & 71 \\
\hline & 94 & 25th & 0.0974 & 92 & 105 & 109 & 0.0678 & 59 & 69 & 72 \\
\hline & 97 & 50th & 0.0970 & 93 & 105 & 110 & 0.0667 & 59 & 69 & 72 \\
\hline & 101 & 75th & 0.0964 & 94 & 106 & 111 & 0.0647 & 60 & 69 & 72 \\
\hline & 107 & 95th & 0.0956 & 95 & 108 & 112 & 0.0623 & 60 & 70 & 72 \\
\hline \multirow[t]{5}{*}{4} & 95 & 5 th & 0.0972 & 93 & 106 & 110 & 0.0674 & 60 & 69 & 72 \\
\hline & 99 & 25th & 0.0967 & 94 & 107 & 111 & 0.0655 & 60 & 70 & 72 \\
\hline & 103 & 50th & 0.0961 & 95 & 108 & 112 & 0.0638 & 60 & 70 & 73 \\
\hline & 108 & 75th & 0.0955 & 96 & 108 & 113 & 0.0619 & 60 & 70 & 73 \\
\hline & 112 & 95th & 0.0949 & 97 & 110 & 114 & 0.0599 & 61 & 70 & 73 \\
\hline \multirow[t]{5}{*}{5} & 101 & 5 th & 0.0964 & 95 & 108 & 112 & 0.0648 & 60 & 70 & 73 \\
\hline & 106 & 25th & 0.0957 & 96 & 109 & 113 & 0.0626 & 61 & 70 & 73 \\
\hline & 109 & 50th & 0.0953 & 97 & 109 & 114 & 0.0612 & 61 & 71 & 73 \\
\hline & 114 & 75th & 0.0946 & 98 & 111 & 115 & 0.0592 & 61 & 71 & 74 \\
\hline & 122 & 95th & 0.0936 & 99 & 112 & 116 & 0.0561 & 61 & 71 & 74 \\
\hline \multirow[t]{5}{*}{6} & 105 & 5 th & 0.0959 & 96 & 109 & 113 & 0.0630 & 61 & 71 & 73 \\
\hline & 112 & 25th & 0.0949 & 98 & 111 & 115 & 0.0601 & 61 & 71 & 74 \\
\hline & 116 & 50th & 0.0944 & 98 & 112 & 116 & 0.0584 & 61 & 71 & 74 \\
\hline & 120 & 75th & 0.0938 & 99 & 113 & 117 & 0.0567 & 62 & 72 & 74 \\
\hline & 127 & 95th & 0.0929 & 101 & 114 & 118 & 0.0542 & 62 & 72 & 75 \\
\hline \multirow[t]{5}{*}{7} & 112 & 5 th & 0.0949 & 98 & 111 & 116 & 0.0598 & 62 & 71 & 74 \\
\hline & 118 & 25th & 0.0941 & 99 & 113 & 117 & 0.0576 & 62 & 72 & 75 \\
\hline & 123 & 50th & 0.0935 & 100 & 114 & 118 & 0.0557 & 62 & 72 & 75 \\
\hline & 126 & 75th & 0.0930 & 101 & 114 & 119 & 0.0544 & 62 & 72 & 75 \\
\hline & 137 & 95th & 0.0916 & 103 & 117 & 121 & 0.0506 & 63 & 73 & 76 \\
\hline \multirow[t]{5}{*}{8} & 112 & 5 th & 0.0949 & 99 & 112 & 116 & 0.0599 & 62 & 72 & 75 \\
\hline & 123 & 25th & 0.0934 & 101 & 114 & 119 & 0.0556 & 62 & 72 & 75 \\
\hline & 128 & 50th & 0.0928 & 102 & 115 & 120 & 0.0538 & 63 & 73 & 76 \\
\hline & 132 & 75th & 0.0922 & 103 & 116 & 121 & 0.0523 & 63 & 73 & 76 \\
\hline & 140 & 95th & 0.0912 & 105 & 118 & 122 & 0.0495 & 63 & 74 & 77 \\
\hline \multirow[t]{5}{*}{9} & 122 & 5 th & 0.0936 & 101 & 115 & 119 & 0.0562 & 63 & 73 & 76 \\
\hline & 127 & 25th & 0.0930 & 102 & 116 & 120 & 0.0543 & 63 & 73 & 76 \\
\hline & 132 & 50th & 0.0923 & 103 & 117 & 121 & 0.0525 & 63 & 73 & 76 \\
\hline & 137 & 75th & 0.0917 & 104 & 118 & 122 & 0.0507 & 63 & 74 & 77 \\
\hline & 145 & 95th & 0.0906 & 106 & 120 & 124 & 0.0479 & 64 & 74 & 77 \\
\hline \multirow[t]{5}{*}{10} & 123 & 5 th & 0.0934 & 102 & 115 & 120 & 0.0556 & 63 & 73 & 76 \\
\hline & 131 & 25th & 0.0924 & 104 & 117 & 121 & 0.0527 & 64 & 74 & 77 \\
\hline & 136 & 50th & 0.0917 & 105 & 118 & 123 & 0.0509 & 64 & 74 & 77 \\
\hline & 141 & 75th & 0.0910 & 106 & 119 & 124 & 0.0491 & 64 & 74 & 77 \\
\hline & 149 & 95th & 0.0901 & 108 & 121 & 125 & 0.0466 & 64 & 75 & 78 \\
\hline \multirow[t]{2}{*}{11} & 129 & 5 th & 0.0927 & 104 & 117 & 122 & 0.0536 & 64 & 74 & 77 \\
\hline & 136 & 25th & 0.0917 & 105 & 119 & 123 & 0.0508 & 64 & 74 & 77 \\
\hline
\end{tabular}


Table 2 Age-height-specific thresholds: 50th, 90th and 95th percentiles of SBP and DBP values for boys aged 3-17 years (Continued)

\begin{tabular}{|c|c|c|c|c|c|c|c|c|c|c|}
\hline \multirow{2}{*}{$\begin{array}{l}\text { Age, } \\
\text { year }\end{array}$} & \multirow{2}{*}{$\begin{array}{l}\text { Height, } \\
\mathrm{cm}\end{array}$} & \multirow{2}{*}{$\begin{array}{l}\text { Height } \\
\text { percentile }\end{array}$} & \multicolumn{4}{|c|}{$\mathrm{SBP}, \mathrm{mmHg}$} & \multicolumn{4}{|c|}{$\mathrm{DBP}, \mathrm{mmHg}$} \\
\hline & & & $\mathrm{S}^{*}$ & $\begin{array}{l}\text { 50th } \\
\text { percentile } \\
\text { (median) }\end{array}$ & $\begin{array}{l}\text { 90th } \\
\text { percentile }\end{array}$ & $\begin{array}{l}\text { 95th } \\
\text { percentile }\end{array}$ & $\mathrm{S}^{*}$ & $\begin{array}{l}\text { 50th } \\
\text { percentile } \\
\text { (median) }\end{array}$ & $\begin{array}{l}\text { 90th } \\
\text { percentile }\end{array}$ & $\begin{array}{l}\text { 95th } \\
\text { percentile }\end{array}$ \\
\hline & 142 & 50th & 0.0910 & 107 & 120 & 124 & 0.0489 & 64 & 75 & 78 \\
\hline & 147 & 75th & 0.0903 & 108 & 121 & 126 & 0.0471 & 65 & 75 & 78 \\
\hline & 156 & 95th & 0.0892 & 110 & 123 & 128 & 0.0443 & 65 & 76 & 79 \\
\hline \multirow[t]{5}{*}{12} & 132 & 5 th & 0.0922 & 105 & 119 & 123 & 0.0522 & 64 & 74 & 77 \\
\hline & 140 & 25th & 0.0912 & 107 & 120 & 125 & 0.0495 & 65 & 75 & 78 \\
\hline & 145 & 50th & 0.0906 & 108 & 121 & 126 & 0.0479 & 65 & 75 & 78 \\
\hline & 151 & 75th & 0.0898 & 109 & 123 & 127 & 0.0460 & 65 & 76 & 79 \\
\hline & 161 & 95th & 0.0886 & 111 & 125 & 129 & 0.0430 & 66 & 76 & 79 \\
\hline \multirow[t]{5}{*}{13} & 133 & 5 th & 0.0921 & 106 & 119 & 124 & 0.0519 & 65 & 75 & 78 \\
\hline & 143 & 25 th & 0.0909 & 108 & 121 & 126 & 0.0487 & 65 & 75 & 78 \\
\hline & 150 & 50th & 0.0900 & 109 & 123 & 127 & 0.0464 & 65 & 76 & 79 \\
\hline & 157 & 75th & 0.0890 & 111 & 125 & 129 & 0.0440 & 66 & 76 & 79 \\
\hline & 164 & 95th & 0.0882 & 112 & 126 & 131 & 0.0420 & 66 & 77 & 80 \\
\hline \multirow[t]{5}{*}{14} & 139 & 5 th & 0.0914 & 108 & 121 & 126 & 0.0499 & 65 & 75 & 78 \\
\hline & 149 & 25th & 0.0901 & 110 & 123 & 128 & 0.0466 & 66 & 76 & 79 \\
\hline & 157 & 50th & 0.0891 & 111 & 125 & 130 & 0.0442 & 66 & 77 & 80 \\
\hline & 164 & 75th & 0.0883 & 113 & 127 & 131 & 0.0422 & 66 & 77 & 80 \\
\hline & 172 & 95th & 0.0872 & 115 & 128 & 133 & 0.0398 & 67 & 78 & 81 \\
\hline \multirow[t]{5}{*}{15} & 145 & 5th & 0.0906 & 109 & 123 & 128 & 0.0479 & 66 & 76 & 79 \\
\hline & 157 & 25th & 0.0891 & 112 & 126 & 130 & 0.0442 & 67 & 77 & 80 \\
\hline & 162 & 50th & 0.0884 & 113 & 127 & 131 & 0.0425 & 67 & 77 & 81 \\
\hline & 168 & 75th & 0.0876 & 114 & 128 & 133 & 0.0408 & 67 & 78 & 81 \\
\hline & 177 & 95th & 0.0866 & 116 & 130 & 135 & 0.0385 & 67 & 78 & 82 \\
\hline \multirow[t]{5}{*}{16} & 153 & 5 th & 0.0896 & 112 & 126 & 130 & 0.0453 & 67 & 77 & 80 \\
\hline & 161 & 25th & 0.0886 & 113 & 127 & 132 & 0.0430 & 67 & 78 & 81 \\
\hline & 168 & 50th & 0.0877 & 115 & 129 & 133 & 0.0410 & 67 & 78 & 81 \\
\hline & 172 & 75th & 0.0871 & 116 & 130 & 134 & 0.0397 & 68 & 78 & 82 \\
\hline & 179 & 95th & 0.0864 & 117 & 131 & 136 & 0.0380 & 68 & 79 & 82 \\
\hline \multirow[t]{5}{*}{17} & 153 & 5 th & 0.0895 & 112 & 126 & 131 & 0.0452 & 67 & 77 & 81 \\
\hline & 165 & 25th & 0.0881 & 115 & 129 & 133 & 0.0419 & 68 & 78 & 81 \\
\hline & 167 & 50th & 0.0878 & 115 & 129 & 134 & 0.0411 & 68 & 78 & 82 \\
\hline & 173 & 75th & 0.0871 & 116 & 130 & 135 & 0.0395 & 68 & 79 & 82 \\
\hline & 180 & 95th & 0.0862 & 118 & 132 & 136 & 0.0377 & 68 & 79 & 82 \\
\hline
\end{tabular}

SBP systolic blood pressure, DBP diastolic blood pressure

$S *$, coefficient of variation of blood pressure

The height is a key covariate associated with BP levels [1]. Indeed, not taking into account the height in establishing the BP references could lead to an inaccurate assessment of the BP in pediatric practice particularly for children who are very small (5th percentile) or very tall (95th percentile). Our BP thresholds do not require the use of height reference tables because it is presented in centimeters. In addition, this presentation of exact height by value (in centimeters) in different categories of height percentile is proposed to the allow evaluation of the children and adolescents BP more convenient and accurate.

We proposed the 90th and 95th BP percentiles to allow the detection of prehypertension and hypertension 
Table 3 Age-height-specific thresholds: 50th, 90th and 95th percentiles of SBP and DBP values for girls aged 3-17 years

\begin{tabular}{|c|c|c|c|c|c|c|c|c|c|c|}
\hline \multirow{2}{*}{$\begin{array}{l}\text { Age, } \\
\text { year }\end{array}$} & \multirow{2}{*}{$\begin{array}{l}\text { Height, } \\
\mathrm{cm}\end{array}$} & \multirow{2}{*}{$\begin{array}{l}\text { Height } \\
\text { percentile }\end{array}$} & \multicolumn{4}{|c|}{$\mathrm{SBP}, \mathrm{mmHg}$} & \multicolumn{4}{|c|}{$\mathrm{DBP}, \mathrm{mmHg}$} \\
\hline & & & $\overline{S *}$ & $\begin{array}{l}\text { 50th percentile } \\
\text { (median) }\end{array}$ & $\begin{array}{l}\text { 90th } \\
\text { percentile }\end{array}$ & $\begin{array}{l}\text { 95th } \\
\text { percentile }\end{array}$ & $\mathrm{S}^{*}$ & $\begin{array}{l}\text { 50th percentile } \\
\text { (median) }\end{array}$ & $\begin{array}{l}\text { 90th } \\
\text { percentile }\end{array}$ & $\begin{array}{l}\text { 95th } \\
\text { percentile }\end{array}$ \\
\hline \multirow[t]{5}{*}{3} & 88 & 5 th & 0.0994 & 93 & 106 & 109 & 0.1313 & 60 & 70 & 73 \\
\hline & 92 & 25th & 0.0996 & 94 & 107 & 110 & 0.1313 & 60 & 70 & 74 \\
\hline & 99 & 50th & 0.0998 & 95 & 108 & 112 & 0.1313 & 60 & 71 & 74 \\
\hline & 103 & 75th & 0.1000 & 96 & 109 & 113 & 0.1313 & 60 & 71 & 74 \\
\hline & 109 & 95th & 0.1002 & 97 & 110 & 114 & 0.1313 & 61 & 71 & 75 \\
\hline \multirow[t]{5}{*}{4} & 95 & 5 th & 0.0984 & 95 & 108 & 111 & 0.1298 & 60 & 71 & 74 \\
\hline & 98 & 25th & 0.0985 & 96 & 108 & 112 & 0.1298 & 61 & 71 & 74 \\
\hline & 102 & 50th & 0.0987 & 97 & 109 & 113 & 0.1298 & 61 & 71 & 74 \\
\hline & 108 & 75th & 0.0989 & 98 & 111 & 114 & 0.1298 & 61 & 72 & 75 \\
\hline & 121 & 95th & 0.0993 & 100 & 113 & 117 & 0.1298 & 62 & 72 & 76 \\
\hline \multirow[t]{5}{*}{5} & 98 & 5th & 0.0973 & 96 & 109 & 113 & 0.1283 & 61 & 71 & 74 \\
\hline & 105 & 25th & 0.0975 & 98 & 110 & 114 & 0.1283 & 61 & 72 & 75 \\
\hline & 110 & 50th & 0.0977 & 98 & 111 & 115 & 0.1283 & 61 & 72 & 75 \\
\hline & 115 & 75th & 0.0979 & 99 & 113 & 117 & 0.1283 & 62 & 72 & 75 \\
\hline & 122 & 95th & 0.0981 & 101 & 114 & 118 & 0.1283 & 62 & 73 & 76 \\
\hline \multirow[t]{5}{*}{6} & 105 & 5 th & 0.0963 & 98 & 111 & 115 & 0.1269 & 62 & 72 & 75 \\
\hline & 113 & 25th & 0.0966 & 100 & 113 & 117 & 0.1269 & 62 & 72 & 76 \\
\hline & 117 & 50th & 0.0967 & 100 & 114 & 118 & 0.1269 & 62 & 73 & 76 \\
\hline & 123 & 75th & 0.0969 & 101 & 115 & 119 & 0.1269 & 62 & 73 & 76 \\
\hline & 131 & 95th & 0.0972 & 103 & 117 & 121 & 0.1269 & 63 & 73 & 77 \\
\hline \multirow[t]{5}{*}{7} & 110 & 5 th & 0.0953 & 100 & 113 & 116 & 0.1254 & 62 & 73 & 76 \\
\hline & 118 & 25th & 0.0955 & 101 & 114 & 118 & 0.1254 & 62 & 73 & 76 \\
\hline & 123 & 50th & 0.0957 & 102 & 116 & 120 & 0.1254 & 63 & 73 & 76 \\
\hline & 128 & 75th & 0.0959 & 103 & 116 & 121 & 0.1254 & 63 & 73 & 77 \\
\hline & 134 & 95th & 0.0961 & 104 & 118 & 122 & 0.1254 & 63 & 74 & 77 \\
\hline \multirow[t]{5}{*}{8} & 117 & 5 th & 0.0943 & 102 & 115 & 118 & 0.1240 & 63 & 73 & 76 \\
\hline & 123 & 25th & 0.0945 & 103 & 116 & 120 & 0.1240 & 63 & 74 & 77 \\
\hline & 128 & 50th & 0.0947 & 104 & 117 & 121 & 0.1240 & 63 & 74 & 77 \\
\hline & 133 & 75th & 0.0948 & 104 & 118 & 122 & 0.1240 & 63 & 74 & 77 \\
\hline & 142 & 95th & 0.0952 & 106 & 120 & 124 & 0.1240 & 64 & 75 & 78 \\
\hline \multirow[t]{5}{*}{9} & 121 & 5 th & 0.0932 & 103 & 116 & 120 & 0.1226 & 63 & 74 & 77 \\
\hline & 129 & 25th & 0.0935 & 104 & 118 & 122 & 0.1226 & 64 & 74 & 77 \\
\hline & 133 & 50th & 0.0937 & 105 & 119 & 123 & 0.1226 & 64 & 74 & 77 \\
\hline & 138 & 75th & 0.0938 & 106 & 120 & 124 & 0.1226 & 64 & 75 & 78 \\
\hline & 149 & 95th & 0.0942 & 108 & 122 & 127 & 0.1226 & 65 & 75 & 78 \\
\hline \multirow[t]{5}{*}{10} & 126 & 5 th & 0.0922 & 104 & 118 & 122 & 0.1212 & 64 & 74 & 77 \\
\hline & 133 & 25th & 0.0925 & 106 & 119 & 123 & 0.1212 & 64 & 75 & 78 \\
\hline & 137 & 50th & 0.0926 & 107 & 120 & 124 & 0.1212 & 64 & 75 & 78 \\
\hline & 143 & 75th & 0.0928 & 108 & 121 & 126 & 0.1212 & 65 & 75 & 78 \\
\hline & 152 & 95th & 0.0931 & 109 & 123 & 128 & 0.1212 & 65 & 76 & 79 \\
\hline \multirow[t]{2}{*}{11} & 127 & 5 th & 0.0911 & 105 & 118 & 122 & 0.1198 & 64 & 75 & 78 \\
\hline & 138 & 25th & 0.0915 & 107 & 121 & 125 & 0.1198 & 65 & 75 & 78 \\
\hline
\end{tabular}


Table 3 Age-height-specific thresholds: 50th, 90th and 95th percentiles of SBP and DBP values for girls aged 3-17 years (Continued)

\begin{tabular}{|c|c|c|c|c|c|c|c|c|c|c|}
\hline \multirow{2}{*}{$\begin{array}{l}\text { Age, } \\
\text { year }\end{array}$} & \multirow{2}{*}{$\begin{array}{l}\text { Height, } \\
\text { cm }\end{array}$} & \multirow{2}{*}{$\begin{array}{l}\text { Height } \\
\text { percentile }\end{array}$} & \multicolumn{4}{|c|}{$\mathrm{SBP}, \mathrm{mmHg}$} & \multicolumn{4}{|c|}{$\mathrm{DBP}, \mathrm{mmHg}$} \\
\hline & & & $\overline{S^{*}}$ & $\begin{array}{l}\text { 50th percentile } \\
\text { (median) }\end{array}$ & $\begin{array}{l}\text { 90th } \\
\text { percentile }\end{array}$ & $\begin{array}{l}\text { 95th } \\
\text { percentile }\end{array}$ & $\overline{S *}$ & $\begin{array}{l}\text { 50th percentile } \\
\text { (median) }\end{array}$ & $\begin{array}{l}90 \text { th } \\
\text { percentile }\end{array}$ & $\begin{array}{l}\text { 95th } \\
\text { percentile }\end{array}$ \\
\hline & 145 & 50th & 0.0917 & 108 & 122 & 127 & 0.1198 & 65 & 76 & 79 \\
\hline & 150 & 75th & 0.0919 & 110 & 123 & 128 & 0.1198 & 65 & 76 & 79 \\
\hline & 157 & 95th & 0.0921 & 111 & 125 & 130 & 0.1198 & 66 & 76 & 79 \\
\hline \multirow[t]{5}{*}{12} & 132 & 5 th & 0.0901 & 107 & 120 & 124 & 0.1184 & 65 & 75 & 78 \\
\hline & 142 & 25th & 0.0905 & 109 & 122 & 126 & 0.1184 & 65 & 76 & 79 \\
\hline & 149 & 50th & 0.0907 & 110 & 124 & 128 & 0.1184 & 66 & 76 & 79 \\
\hline & 154 & 75th & 0.0908 & 111 & 125 & 129 & 0.1184 & 66 & 76 & 79 \\
\hline & 161 & 95th & 0.0911 & 112 & 126 & 131 & 0.1184 & 66 & 77 & 80 \\
\hline \multirow[t]{5}{*}{13} & 139 & 5 th & 0.0892 & 109 & 122 & 126 & 0.1171 & 65 & 76 & 79 \\
\hline & 148 & 25th & 0.0895 & 110 & 124 & 128 & 0.1171 & 66 & 76 & 79 \\
\hline & 154 & 50th & 0.0897 & 111 & 125 & 130 & 0.1171 & 66 & 77 & 80 \\
\hline & 159 & 75th & 0.0899 & 112 & 126 & 131 & 0.1171 & 66 & 77 & 80 \\
\hline & 166 & 95th & 0.0901 & 114 & 128 & 133 & 0.1171 & 67 & 77 & 80 \\
\hline \multirow[t]{5}{*}{14} & 143 & 5 th & 0.0882 & 110 & 123 & 128 & 0.1157 & 66 & 76 & 79 \\
\hline & 153 & 25th & 0.0885 & 112 & 125 & 130 & 0.1157 & 66 & 77 & 80 \\
\hline & 157 & 50th & 0.0887 & 113 & 126 & 131 & 0.1157 & 67 & 77 & 80 \\
\hline & 162 & 75th & 0.0888 & 113 & 128 & 132 & 0.1157 & 67 & 77 & 80 \\
\hline & 168 & 95th & 0.0890 & 115 & 129 & 134 & 0.1157 & 67 & 78 & 81 \\
\hline \multirow[t]{5}{*}{15} & 147 & 5 th & 0.0872 & 111 & 125 & 129 & 0.1144 & 67 & 77 & 80 \\
\hline & 154 & 25th & 0.0874 & 113 & 126 & 131 & 0.1144 & 67 & 77 & 80 \\
\hline & 159 & 50th & 0.0876 & 113 & 127 & 132 & 0.1144 & 67 & 77 & 80 \\
\hline & 164 & 75th & 0.0878 & 114 & 128 & 133 & 0.1144 & 67 & 78 & 81 \\
\hline & 171 & 95th & 0.0880 & 116 & 130 & 135 & 0.1144 & 68 & 78 & 81 \\
\hline \multirow[t]{5}{*}{16} & 148 & 5th & 0.0862 & 112 & 125 & 130 & 0.1131 & 67 & 77 & 80 \\
\hline & 156 & 25th & 0.0864 & 114 & 127 & 131 & 0.1131 & 67 & 77 & 80 \\
\hline & 161 & 50th & 0.0865 & 114 & 128 & 133 & 0.1131 & 68 & 78 & 81 \\
\hline & 164 & 75th & 0.0867 & 115 & 129 & 133 & 0.1131 & 68 & 78 & 81 \\
\hline & 174 & 95th & 0.0870 & 117 & 131 & 136 & 0.1131 & 68 & 78 & 81 \\
\hline \multirow[t]{5}{*}{17} & 150 & 5th & 0.0851 & 113 & 126 & 131 & 0.1118 & 67 & 77 & 80 \\
\hline & 156 & 25th & 0.0853 & 114 & 128 & 132 & 0.1118 & 68 & 78 & 81 \\
\hline & 162 & 50th & 0.0855 & 115 & 129 & 133 & 0.1118 & 68 & 78 & 81 \\
\hline & 166 & 75th & 0.0856 & 116 & 130 & 134 & 0.1118 & 68 & 78 & 81 \\
\hline & 173 & 95th & 0.0858 & 117 & 131 & 136 & 0.1118 & 68 & 79 & 82 \\
\hline
\end{tabular}

SBP systolic blood pressure, $D B P$ diastolic blood pressure

$S *$, coefficient of variation of blood pressure

in children and adolescents. These cut-off values for prehypertension and hypertension respectively, were used according to the criteria's definition of the Fourth Report on the Diagnosis, Evaluation and Treatment of High Blood Pressure in Children and Adolescents [1] and to the recommendations of the European Society of Hypertension on the Management of High Blood Pressure in Children and Adolescents [2]. Because of the large amount of data available, the Task Force for Blood Pressure in Children [1] is still the study of reference. We have not presented the 99th BP percentile. Indeed, a child or adolescent with BP value that defines hypertension ( $\geq$ 95th percentile compared to reference tables) will not be diagnosed by this measure alone as being hypertensive but other additional BP measures are recommended on different occasions $[1,2]$. Owing to 


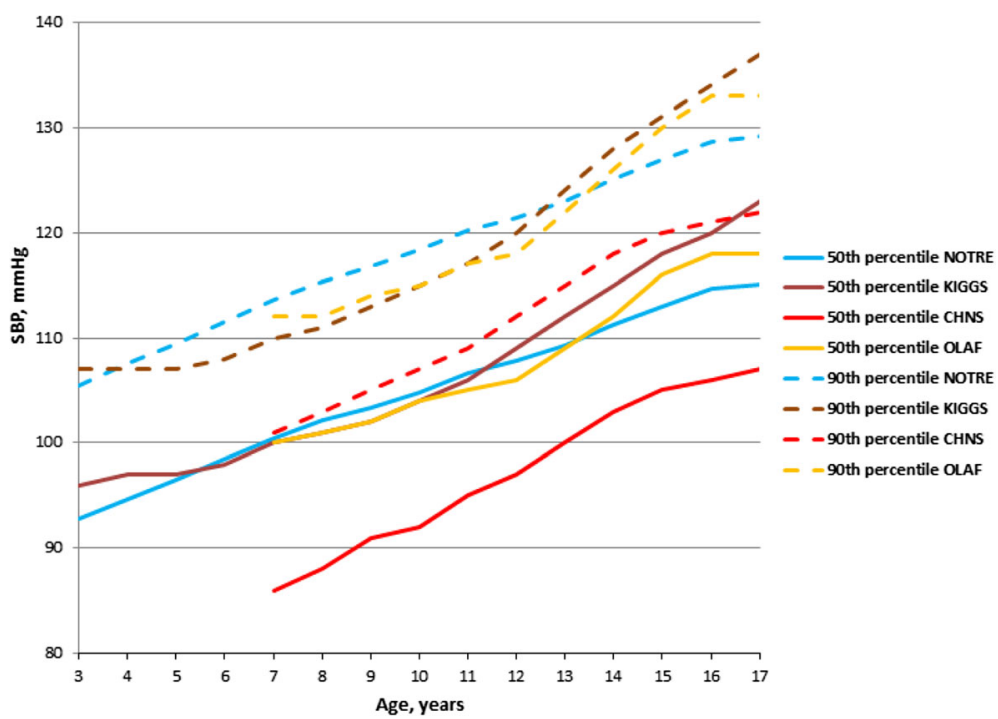

Fig. 1 The 50th and 90th percentiles of SBP for the median height for our study (NOTRE) compared with the German (KIGGS), Polish (OLAF) and Chinese (CHNS) boys

cultural and ethnic diversity of the peoples of the DRC, our results cannot extrapolate to the entire nation.

The 90th SBP percentile for the median height of adolescents for boys aged 11-17 years and girls aged 10-17 years, respectively of $120-132 \mathrm{mmHg}$ and $120-131 \mathrm{mmHg}$, was equal to or higher than the threshold $120 \mathrm{mmHg}$ for the identification of prehypertension as recommended by the fourth report of the National High Blood Pressure Education Program (NHBPEP) Working Group on High Blood Pressure in Children and Adolescents [1], the European Society of Hypertension [2] and the Seventh Report of the Joint National Committee on Prevention, Detection, Evaluation, and treatment of High Blood Pressure (JNC VII)
[24]. This observation may indicate the need for careful consideration of changes to the definition of prehypertension in the case of adolescent. The fact that the BP of Congolese children of Lubumbashi is so high at the beginning of adolescence, could be justified by several observations showing that hypertension in black subjects occurs at the early age $[25,26]$. In addition, the 90th BP percentile for adolescents aged 17 years (in both sexes) was $130 \mathrm{mmHg}$, which is equal to the recommended optimal SBP for adult to define metabolic syndrome [27]. Our results are almost similar to those reported by Kulaga et al. [15] and Neuhauser et al. [14]. For the first authors, the 90th SBP percentile for adolescents age range 13-17 years

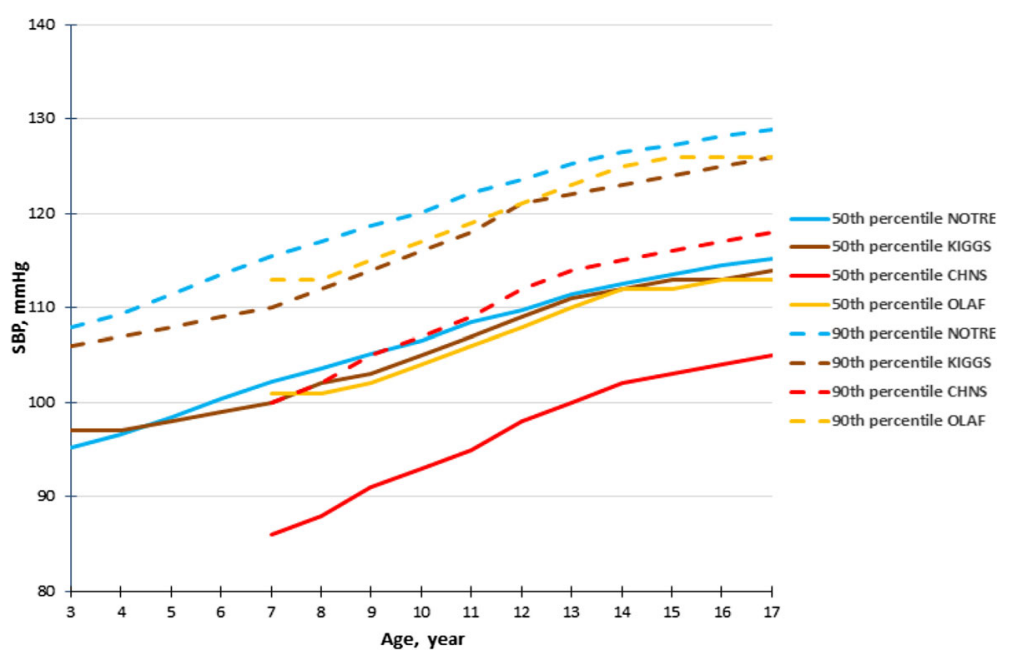

Fig. 2 The 50th and 90th percentiles of SBP for the median height for our study (NOTRE) compared with the German (KIGGS), Polish (OLAF) and Chinese (CHNS) girls 


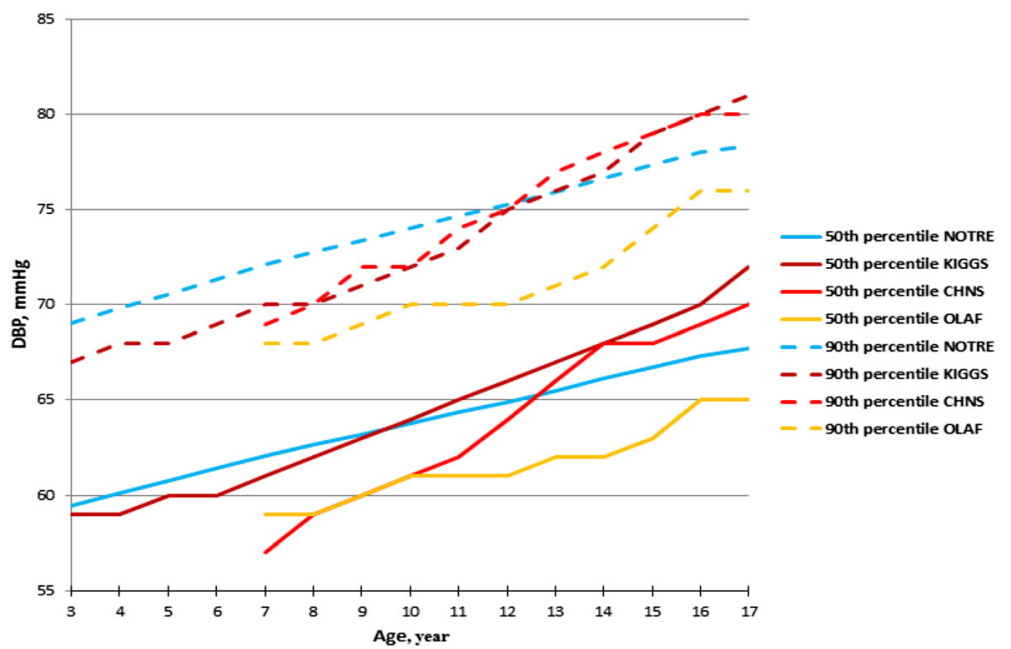

Fig. 3 The 50th and 90th percentiles of DBP for the median height for our study (NOTRE) compared with the German (KIGGS), Polish (OLAF) and Chinese (CHNS) boys

(boys) and 12-17 years (girls) was respectively 122$133 \mathrm{mmHg}$ and 120-126 $\mathrm{mmHg}$. While for the second author, adolescents aged 12 to 17 (both boys and girls) had their 90th SBP percentile respectively 120-137 $\mathrm{mmHg}$ and 122-126 mmHg. In the CHNS study [16], only adolescent boys aged 15 to 17 had the 90th percentile of SBP and DBP at 122 and $80 \mathrm{mmHg}$ close to the threshold of $120 / 80 \mathrm{mmHg}$ for the identification of prehypertension. In this study the BP values are examined by mercury sphygmomanometer.

We compared our percentiles with the KIGGS [14], OLAF [15] and CHNS [16] studies because they are all tools that aim to solve the same problem: screening and detection of elevated $\mathrm{BP}$ in children. In addition, the construction of the BP percentiles was based on the normal weight subjects and developed simultaneously by age and height.

Several reasons could explain the differences with each study. In particular, the devices used for the BP measurement (auscultatory, oscillometric). It is known as oscillometric devices provide high values of the BP compared to mercury sphygmomanometer [1]. The Datascope Accutor Plus had passed the standards of the Association for the

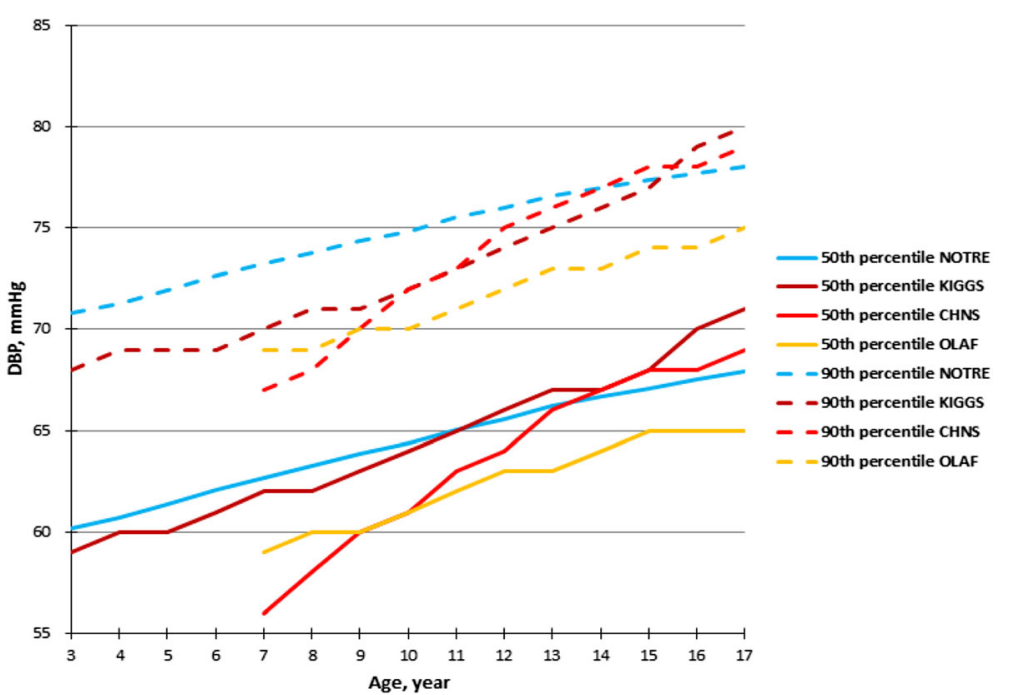

Fig. 4 The 50th and 90th percentiles of DBP for the median height for our study (NOTRE) compared with the German (KIGGS), Polish (OLAF) and Chinese (CHNS) girls 
Advancement of Medical Instrumentation [28] and the British Hypertension Society [29] for adults and had been validated in children aged 5 to 15 years compared to the mercury sphygmomanometer as required of the International Protocol of the European Society of Hypertension (ESH-IP) [30]. In the validation study in children [21], measures of the Datascope Accutor Plus were close to the sphygmomanometer measurements: the mean (SD) of the differences was for PAS values (oscillometric least auscultation) of - 0.9 (4.33) $\mathrm{mmHg}$ and DBP - 1.2 (6.48) $\mathrm{mm} \mathrm{Hg}$. The number of the BP measurements used for the establishment of the BP percentiles. As for Kulaga et al. [15], our study used the mean of the last two measures (of the three) BP for statistical analysis because the first one are often high $[31,32]$. The statistical method used for the BP percentiles construction. The GAMLSS method was used in our study and those of Neuhauser et al. [14] et Yan et al. [16]; while Kulaga et al. [15] had used the polynomial regression. Another possible reason is the lack of a uniform definition for overweight and obesity for the noninclusion of this group of children and adolescents in the study of normal weight population. Neuhauser et al. [14] had used the 90th percentile of the BMI of the German reference and Yan et al. [16] were based on the reference BMI of Chinese children and adolescents. Like in the OLAF study [15], we used the definition IOTF [17], because we have not BMI reference for children in our country and it is consistent with the levels of overweight and obesity in childhood and adolescence (2-18 years), with the definition of overweight $(\geq 25 \mathrm{~kg} / \mathrm{m} 2)$ and obesity $(\geq 30 \mathrm{~kg} / \mathrm{m} 2)$ in adults. We also took into account the recommendations of the European Childhood Obesity Group [33]: which suggest the use of the definition of the IOTF or the WHO definition in epidemiological studies. Ethnic, racial, geographic differences may also explain the variability of the $B P$ in the populations studied $[25,34]$.

A possible limitation of our study is the selection bias. Owing to the lack of official documents, we used a reported age declaration by parents or guardians. Another limitation was related to a lack external validation made to assess the performance of our proposed BP thresholds.

\section{Conclusion}

We established, for the first time, the thresholds percentiles (50, 90 and 95) of the BP for specific age and height of children and adolescents aged 3 to 17 years in Lubumbashi (DRC) for the use in pediatric clinical practice. Early identification of prehypertension and hypertension in children and adolescents leads to early action to the support and possibly the prevention of late morbidity and mortality. The BP thresholds percentiles proposed by the current study enable to develop a local program of health promotion in schools and family.
We observed that the 90th percentile of SBP in early adolescence is high and this corresponds to the prehypertension thresholds requiring further studies.

\section{Additional file}

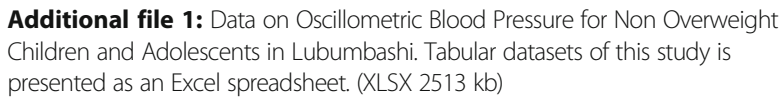

Additional file 1: Data on Oscillometric Blood Pressure for Non Overweight Children and Adolescents in Lubumbashi. Tabular datasets of this study is presented as an Excel spreadsheet. (XLSX 2513 kb)

\begin{abstract}
Abbreviations
BCCE: Box-Cox and Cole Green; BCPE: Box-Cox power exponential; BMI: Body mass index; BP: Blood pressure; CHNS: China Health and Nutrition Survey; DBP: Diastolic blood pressure; DRC: Democratic Republic of Congo; ESH: European Society of Hypertension; GMLSS: Generalized Additive Models for Location, Shape, and Scale; IOTF: International Obesity Task Force; IPESH: International Protocol of the European Society of Hypertension; JNC VII: Seventh Report of the Joint National Committee on Prevention, Detection, Evaluation, and treatment of High Blood Pressure; KIGGS: German Health Interview and Examination Survey for Children and adolescents; LMS: Lambda Mu Sigma; NHBPEP: National High Blood Pressure Education Program Working Group on High Blood Pressure in Children and Adolescents; OLAF: Elaboration of the reference range of arterial blood pressure for the population of children and adolescents in Poland; SBP: Systolic blood pressure; SD: Standard deviation; SPSS: Statistical Package for Social Sciences
\end{abstract}

Acknowledgements

We thank Dr. Robert A. Rigby for his kind help in modeling blood pressure using GAMLSS program.

\section{Funding}

We have not received any funding. The study was conducted on our own.

Availability of data and materials

Raw data and other relevant materials are available upon request from the corresponding author (OM).

\section{Authors' contributions}

EKM, CKM, JMM, PKK, CNK, OM, DNN and WY carried out the conceptualization, design, data collection and analysis for the study. ONL, JKK, FKM and OM contributed to the interpretation of the findings and the drafting of the article. EKM and OM wrote the manuscript. All authors read and approved the final manuscript.

\section{Ethics approval and consent to participate}

The approval to conduct the study and authorizations were obtained from the Medical Ethic Committee of the University of Lubumbashi (UNILU/CEM/ 027/2013-27 september 2013), the Provincial Ministry of Education, Scientific Research, Transport and Energy of Katanga (N 10.5/0209/CAB/MIN.PROV/ ED.R.TE/KAT/2014-11 march 2014) and the authorities of the selected schools. Data was used with high confidentiality and no names were recorded. The informed written consent to participate was obtained from the parent/guardians of the child or adolescent.

\section{Consent for publication}

The informed written consent to participate and for the publication of individual clinical details was obtained from the parent/guardians of the child or adolescent.

\section{Competing interests}

The authors declare that they have no competing interests.

\section{Publisher's Note}

Springer Nature remains neutral with regard to jurisdictional claims in published maps and institutional affiliations. 


\section{Author details}

${ }^{1}$ Department of Internal Medicine, Sendwe Hospital, University of Lubumbashi, Lubumbashi, Democratic Republic of Congo. ${ }^{2}$ Department of Public Health, University of Lubumbashi, Lubumbashi, Democratic Republic of Congo. ${ }^{3}$ Department of Internal Medicine, University Clinic, University of Lubumbashi, Lubumbashi, Democratic Republic of Congo. ${ }^{4}$ Department of Pediatrics, University Clinic, University of Lubumbashi, Lubumbashi, Democratic Republic of Congo. ${ }^{5}$ School of Public Health, University of Lubumbashi, Lubumbashi, Democratic Republic of Congo. ${ }^{6}$ Department of Research, Higher Institute of Medical Techniques, Lubumbashi, Democratic Republic of Congo. ${ }^{7}$ Department of Gynecology, Clinical University of Lubumbashi, University of Lubumbashi, Lubumbashi, Democratic Republic of Congo. ${ }^{8}$ Department of Clinical Epidemiology, Children's Hospital of Fudan University, 399 Wanyuan Road, Shanghai 201102, China.

Received: 23 August 2016 Accepted: 2 January 2018

Published online: 19 January 2018

\section{References}

1. NHBPEP. The fourth report on the diagnosis, evaluation, and treatment of high blood pressure in children and adolescents. Pediatrics. 2004; 114(2):555-76

2. Lurbe E, Cifkova R, Cruickshank JK, Dillon MJ, Ferreira I, Invitti C, et al. Management of high blood pressure in children and adolescents: recommendations of the European Society of Hypertension. J Hypertens. 2009:27(9):1719-42.

3. Falkner B. Recent clinical and translational advances in pediatric hypertension. Hypertension. 2015;65:926-31.

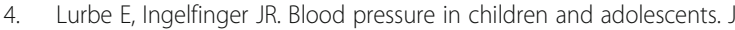
Hypertens. 2016;34(2):176-83.

5. Brady TM, Fivush B, Flynn JT, Parekh R. Ability of blood pressure to predict left ventricular hypertrophy in children with primary hypertension. J Pediat 2008;152(1):73-8.

6. Reinehr T, Kiess W, De Sousa G, Stoffel-Wagner B, Wunsch R. Intima media thickness in childhood obesity: relations to inflammatory marker, glucose metabolism, and blood pressure. Metabolism. 2006;55(1):113-8.

7. Chen X, Wang Y. Tracking of blood pressure from childhood to adulthood: a systematic review and meta-regression analysis. Circulation. 2008;117(25):3171-80.

8. Ke L, Brock KE, Cant RV, Li Y, Morrell SL. The relationship between obesity and blood pressure differs by ethnicity in Sydney school children. Am J Hypertens. 2008;22(1):52-8.

9. Marras AR, Bassareo PP, Ruscazio M. The prevalence of paediatric hypertension, emphasising the need to use specific population references: the Sardinian hypertensive adolescents research Programme study. Cardiol Young. 2009;19(3):233-8.

10. Kotchen TA. Obesity-related hypertension: epidemiology, Pathophysiology, and clinical management. Am J Hypertens. 2010;23(11):1170-8.

11. Nguyen T, Lau DCW. The obesity epidemic and its impact on hypertension. Can J Cardiol. 2012;28:326-33.

12. Barlow SE, Dietz WH. Obesity evaluation and treatment: expert committee recommendations. Pediatrics. 1998;102(3):e29.

13. Dong B, Ma J, Wang HJ, Wang ZQ. The association of overweight and obesity with blood pressure among chinese children and adolescents. Biomed Environ Sci. 2013;26(6):437-44. https://doi.org/10.3967/08953988.2013.06.004

14. Neuhauser HK, Thamm M, Ellert U, Hense HW, Rosario AS. Blood pressure percentiles by age and height from nonoverweight children and adolescents in Germany. Pediatrics. 2011:127:e978-88.

15. Kulaga Z, Litwin M, Grajda A, Kułaga K, Gurzkowska B, Gozdz M, et al. Oscillometric blood pressure percentiles for polish normal-weight schoolaged children and adolescents. J Hypertens. 2012;30(10):1942-54.

16. Yan W, Liu F, Li X, Wu L, Zhang Y, Cheng Y, et al. Blood pressure percentiles by age and height for non-overweight Chinese children and adolescents: analysis of the China health and nutrition surveys 19912009. BMC Pediatr. 2013;13:195

17. Cole TJ, Bellizzi MC, Flegal K, Dietz WH. Establishing a standard definition for child overweight and obesity worldwide: international survey. BMJ. 2000;320:1240-3.

18. Cole TJ, Green PJ. Smoothing reference centile curves: the LMS method and penalized likelihood. Stat Med. 1992;11(10):1305-19.
19. Rigby RA, Stasinopoulos DM. Using the box-Cox t distribution in GAMLSS to model skewness and kurtosis. Stat Model. 2006:6:209-29.

20. Cole TJ, Stanojevic S, Stocks J, Coates L, Hankinson JL, Wade AM. Age- and size-related reference ranges: a case study of spirometry through childhood and adulthood. Statist Med. 2009;28:880-98.

21. Wong SN, Sung RYT, Leung LCK. Validation of three oscillometric blood pressure devices against auscultatory mercury sphygmomanometer in children. Blood Press Monit. 2006;11(5):281-91.

22. Sung YTR, Choi KC, So HK, Nelson EAS, Li AM, Kwok CWL, et al. Oscillometrically measured blood pressure in Hong Kong Chinese children and associations with anthropometric parameters. J Hypertens. 2008;26(4):678-84

23. Sorof JM, Alexandrov AV, Cardwell G, Portman RJ. Carotid artery intimalmedial thickness and left ventricular hypertrophy in children with elevated blood pressure. Pediatrics. 2003;111:61-6.

24. Chobanian AV, Bakris GL, Black HR, Cushman WC, Green LA, Izzo JL, et al. The seventh report of the joint National Committee on prevention, detection, evaluation, and treatment of high blood pressure: the JNC 7 report. JAMA. 2003;289:2560-72.

25. Amah G, Lévy BI. The specificities of hypertension in black African subjects. Sang Thrombose Vaisseaux. 2007;19(10):519-25. https://doi. org/10.1684/stv.2008.0213.

26. Still CH, Craven TE, Freedman BI, Van Buren PN, Sink KM, Killeen AA. Baseline characteristics of African Americans in the systolic blood pressure intervention trial. J Am Soc Hypertens. 2015;9(9):670-9.

27. Alberti KGMM, Eckel RH, Grundy SM, Zimmet PZ, Cleeman Jl, Donato KA, et al. Harmonizing the metabolic syndrome: a joint interim statement of the international diabetes federation task force on epidemiology and prevention; National Heart, Lung, and Blood Institute; American Heart Association; World Heart Federation; International. Circulation. 2009;120:1640-5.

28. Anwar YA, Tendler BE, McCabe EJ, Mansoor GA, White WB. Evaluation of the Datascope Accutorr plus according to the recommendations of the Association for the Advancement of medical instrumentation. Blood Press Monit. 1997:2(2):105-10.

29. White WB, Herbst T, Thavarajah S, Giacco S. Clinical evaluation of the Trimline blood pressure cuffs withthe Accutorr plus monitor. Blood Press Monit. 2003;8(3):137-40.

30. O'Brien E, Pickering T, Asmar R, Myers M, Parati G, Staessen J, et al. Working group on blood pressure monitoring of the European Society of Hypertension International Protocol for validation of blood pressure measuring devices in adults. Blood Press Monit. 2002;7(1):3-17.

31. Park MK, Menard SM. Normative oscillometric blood pressure values in the first 5 years in an office setting. Am J Dis Child. 1989:143(7):860-4. https:// doi.org/10.1001/archpedi.1989.02150190110034.

32. Gillman MW, Cook NR. Blood pressure measurement in childhood epidemiological studies. Circulation. 1995;92(4):1049-57.

33. Rolland-Cachera MF. Childhood obesity: current definitions and recommendations for their use. Int J Pediatr Obes. 2011;6:325-31.

34. Rosner B, Cook N, Portman R, Daniels S, Falkner B. Determination of blood pressure percentiles in normal-weight children: some methodological issues. Am J Epidemiol. 2008;167(6):653-66.

\section{Submit your next manuscript to BioMed Central and we will help you at every step:}

- We accept pre-submission inquiries

- Our selector tool helps you to find the most relevant journal

- We provide round the clock customer support

- Convenient online submission

- Thorough peer review

- Inclusion in PubMed and all major indexing services

- Maximum visibility for your research

Submit your manuscript at www.biomedcentral.com/submit 